\title{
An Overview and Perspective of Desktop Grid Middleware to Speed up Smul8 Process by Predicting Child Mortality Birth and Death Rate in West African Countries
}

\author{
Edje E. Abel \\ Department of Mathematics \\ and Computer Science \\ Delta State University, Abraka \\ P.M.B. 01 \\ Delta State, \\ Nigeria
}

\begin{abstract}
Desktop Grid middleware enables the connection of two or more computer systems to work together by harnessing their processing power to perform complex computational task. Desktop Grid Computing is fault tolerant due to the fact that, the failure of one computer system does not halt the execution process of the assigned task. This research study explore in detail about desktop grid computing and computer simulation. It discuss briefly about the child mortality birth and death rate in West African countries. The challenges of the existing systems used to predict child mortality birth and death rate in West African States were highlighted; thus a proposed system solution called "Desktop Grid Middleware to Speed up Smul8" was modeled to resolve some of the flaws of the existing system. The responsibility of the proposed system is to speed up the execution process via Simul8 to predict the total number of child mortality birth and death rate, within a specified timeframe (e g. from the year 2016 to 2090) at the shortest possible time by combining the processing powers of four Computer Systems. The proposed system solution was also recommended to health agencies and other humanitarian agencies and end with a conclusion.
\end{abstract}

\section{Keywords}

Monte Carlo Simulation (MCS) , Simul8 Software Application (SSA), Nations inter-agency group for Mortality Estimation (UNIGME), Desktop Grid Middleware (DGM), Hydron Collider (HC), Discrete Event-type Simulation (DES) and Dynamic or Continuous Simulation (DCS)

\section{INTRODUCTION}

The emergence of Desktop grid middleware has impacted positively in business organizations, health sector and scientific research purposes. According to [27], Grid computing is a model of distributed computing that uses geographically and administratively disparate resources that are found on the network. These resources include processing power, storage facilities and processing speed e $\mathrm{t} \mathrm{c}$. The idea of grid computing is to harness the idle processing power of computer systems on a network to speed up the execution of specific task. The structure of grid computing system is often based on a central system splitting a given tasks or job into fragments, thereby dispatching them to other work stations to execute. The main benefit of grid computing is that, jobs are executed within a limited timeframe. Also, it speed up simulation processes that deals on forecasting weather conditions, profits to be made in a business firm and other intensive scientific computational tasks e t $\mathrm{c}$.

\section{GRID COMPUTING}

Grid computing is a hardware and software infrastructure that enables the integrated and collaborative use of computers and networks owned and managed by multiple organizations [5]. This means that authorized end-Users can access resources that reside on the interconnected networks of organizations from remote computers. It is said that Grid computing uses some elements of the internet (such as the internet protocol) to actualize its connection. The internet is about getting computers to talk together while grid computing is about getting computers to work together [10]. There are different types of grid computing middleware which are called Globus, Boinc, Condor and Legion e t c. The most widely used is the globus middleware. The globus grid middleware is a multiinstitutional research effort to create a basic infrastructure and high level services for a computational grid [19]. This involves an infrastructure for resource sharing (such as hardware, and applications) within two or more organizations. These middleware grids enable high productivity by increasing the average computational performance available to important applications "regardless of the spatial distribution of both resources and users" [19].

\subsection{Desktop Grid Computing}

Desktop grid computing is a computing paradigm that achieves a high throughput computing by harvesting a number of idle computing resources on the internet [39]. These resources (such as database application, processing speed, storage capability etc.) may be part of similar Local Area Networks that are interconnected globally over the internet. [11] argues that the power of network storage and computing resources is projected to double every 9,12 and 18 months respectively; which represents enormous computing resources to the public. This could result to software applications having non-trivial processing specifications to run faster using available computer resources.

According to [14], desktop grid computing has achieved tremendous computing power with low cost, through opportunistic sharing to exploit large collections of personal computers and workstations across the internet. It is said that 
desktop grid computing middleware consist of the master, manager worker architecture. Whereby, "a trusted master supplies jobs to a set of volunteer workers via the manager" [42].

According to [39], desktop grid computing environment mainly comprises of clients, volunteers and volunteer server. In our own case, the client can be express as a master, while the volunteers and volunteer server may be workers and manager. The master sends job and the workers (volunteer) is a donator that is willing to share its computing resources. The manager (server) is responsible for controlling workers and jobs send by the master. For instance, when a master submit job to the manager, thus the task is divided into sub task by the manager and dispatch the sub-divided tasks to workers [27]. Each volunteers or workers performs its own share of task and return a result to the manager, which in turn pass the result to the master.

An important feature of desktop grid computing is decentralization control and management of resources. It allows resources provisioning, utilization and reconfiguration without the intersection by centralized management [13]. This means that all networks that are interconnected to form the desktop grid environment can maintain their own resources. They do not have to rely on a centralized based system and resources (such as date, software application and processing) to perform jobs. Centralized system are not hundred percent reliable because data or resources could be loss due to system failure [13].

\subsection{Types of Desktop Grid Computing}

There are three main types of desktop Grid Computing which includes Global, Local and Commercial Desktop Grid. Global Desktop Grid (also known as public Desktop Grids) consists of a publicly accessible server providing services to clients [1]. Clients can use computers to request for information from the server at anytime, anywhere and there is no assurance that a client which started the computation will finish the computational process. [25], describes commercial enterprise desktop grid as infrastructure that is confined to an institutional boundary. The idleness of processing capacity of an enterprise's personal computers is used to support the execution of the enterprise's applications. These computer systems could be scattered all over various departments within the enterprise company. An enterprise could be a large company that has branches all over the world; with multiple interconnected computer networks, or a small company residing in one building with a single local area network.

Local desktop grid is especially used for businesses. They tend not to send out application or data to un-trusted third parties (an outside or the world). This is achievable when the "project and clients are shielded from the world by firewalls and other network security measures" [23]. This enables clients to access local resources securely and flexible. Performance is more likely to be predictable since the resources are not voluntarily offered. There are quit similarity between commercial enterprise and local desktop grid. This was argued by [15], which said that both are intended for institutional and industrial use.

\section{COMPUTER SIMULATION}

Computer simulation is used to determine the challenges or risks that are prevalent with new systems or updating an existing system. Presently, organizations always want to be assured that investment in the acquisition of ICT facilities meet expected result. [20], Simulation is the use of computer program to model a real world system, in order to validate decisions affecting the system. It can also be describe as a technique used for emulating the behavior of a specific event or system by means of a model, in other to actualize information more precisely or to train personnel more effectively and efficiently. A computer based simulation is a program that embodies some aspect of the world, allows the user to make inputs to the model, and displays the result [36]. In order words, it emulates real event or system and provides the opportunity to make changes either to upgrade the system performance or to make correction. According to [35], Computer simulation is used to reduce the risk associated with creating new systems or making changes to existing ones.

[26] defined computer simulation as the manipulation of a model in such a way that it operates on time or space to compress it, thus enabling one to perceive the interactions that would not otherwise be apparent because of their separation in time or space. In other words, it can be used to determine the interactions that occur between system components that would not be visible due to their separation in time and space, with the aid of application software. Modeling on the other hand is used to represent a system as specific point in time with the aid of objects in other to enhance understanding of the real system.

Computer simulation tends to impact positively in every sector such as the educational, business firms and health Government e t c. According to [41] software development organizations has benefited tremendously in the used of programmed simulation to improve project management, training and enhancing strategic management software development. Also, it enables software designers to characterize or visualize a system that is yet to exist, so as to achieve an optimum solution. For instance, "using computer simulation to model manufacturing facility for experiment, with layout of different capacity machines and storage bins, for preparation and transfer of material, so as to improve efficiency" [26]. In the educational sector, computer simulation allows researchers to achieve accurate and analytic techniques that may be use for design and analyzing a system. In order words, computer simulations are used as a "research tools to establish trends, demonstrate relationship between system parameters or make predictions about the future" [12]. There are different kinds of simulation methods which includes dynamic, discrete event and static simulation. Dynamic or continuous simulation technique is used to simulate an event or systems in which the variables changes continuously [29]. Dynamic simulations are mostly use for the predictions of rocket trajectories, hydrogen bomb dynamics, electric circuit simulation and flight testing exercise or event $\mathrm{e}$ $\mathrm{t} \mathrm{c}$. Discrete event simulation is when the variable of a system or event changes simultaneously over a specific discrete period of time and discrete steps or procedures [8]. Good examples of discrete event simulation are the forecasting of weather condition, predicting the population of humans residing in a specific geographical location e $\mathrm{t}$ c. Static Simulation takes a snapshot at a single point in time e $g$. Monte Carlo simulation. It captures events that must be sensitized waiting to be executed in the future.

Monte Carlo simulation is a type of simulation that relies on repeated random sampling and statistical analysis to compute the results [37]. It is often use as a method of executing what software programmers called What-If analysis or event, whereby a set conditions must be met before they are 
executed. However, [3] stated that "Monte Carlo methods are extensively used in financial institutions to compute European option prices, to evaluate sensitivities of portfolios to various parameters and to compute risk measurements". It is mostly implemented on Microsoft Excel worksheet and has been used to simulate diverse real world events and complex computational systems such as Grid Computing e t c. MonteCarlo simulation was applied to the large Hydron Collider (developed in Geneva) grid in order to allow scientist around the world analyze data from the proto-proton collision [14]. It is said that the data collected from the collision will enable scientist to know the origin of mass in the universe as well as discovery new spatial dimensions.

Simul8 is a computer software application that is use for discrete event simulation. It allows the user to create a visual model of the system being investigated by drawing objects directly on the screen [17]. It consist of working tools which are displayed by animation on the screen so that the "correctness of the model can be assessed before simulation can be undertaken" [6]. According to [20], as an objectoriented modeling tool, Simul8 incorporates programming language and model visualization capabilities that enables it to create accurate flexible and robust simulations more rapidly. Simul8 has been use successfully to simulate most aspect of real life discrete event systems. [25] carried out an experiment by using Simul8 application package to simulate the number of airplanes taken off and landing at a specific time frame, at Heathrow Airport London; by speeding the process with the aid of Desktop Grid Computing. The out-come of the experiment shows that desktop grid can actually speed up the process of simul 8

\section{CHILD MORTALITY BIRTH AND Death RATE IN WEST AFRICAN COUNTRIES}

Child mortality rate in Africa is in the increase compared to any other continent. Particularly, West African countries experience child mortality two to three times higher than neighboring countries in the Northern and Southern African [9]. Several factors have been acknowledged as the basis of child survival at birth. These include environmental factors, maternal sensitization, and socioeconomic factor e t c. Child death rate is caused by premature and low birth weight infants, diseases, delivery complications and the pace of child bearing. In particular, short preceding birth intervals are believed to increase infant's risk of mortality because the mother's nutritional reserves have not fully recovered from previous birth [9]. [40], conceived that most child mortality death is cause by several diseases which are environmental oriented. These include poor sewage disposal system, inadequate supply of clean drinkable water are a major causes of typhoid, malarial, and cholera which are prevalent in the West African sub-region.

According to [7], in 20126.6 million children died before reaching their fifth birthday, mostly from preventable causes and treatable diseases, even though the knowledge and technologies for life-saving interventions are available. Research shows that 15 percent of the 6.6million death, is from West African sub -region. The latest data from World Health Organization (WHO) in the year 2013 shows that West Africa has the world's highest under five mortality rates. West African region comprises of twelve six countries which includes Nigeria, Ghana, Chad, Benin Republic, Cameroun, Niger Togo e t c. Record shows that infant under the age of five mortality rate stands at 100 or more deaths per 1,000 live births are in West Africa Region [44]. In other words, one out of every eleven children born in West Africa dies before the age of 5 . This is nearly 15 times the average rate ( 1 in 159) in high- income countries (REF).

Due to the incessant child mortality death rate in the region, Government agencies from this region and some NGO's have come together to find a means of monitoring and preventing the scourge. United Nations inter-agency group for Mortality Estimation (UNIGME) was formulated in 2014 to share data on child mortality, harmonize estimates within the UN system, and improve methods in other to enhance country capacity to produce timely assessed estimates of child Mortality death rate [7]. Predicting Child mortality death and birth rate requires a system for counting the number of live births. Presently, only one in eight of the world's deaths and births are accounted for. Also, the causes of death are identified and recorded accurately. Most developing countries use surveys of a limited sample of households to produce child mortality birth and death statistics. West African countries Lack vital registration system which leads to inadequate child mortality death rate information to support sound public health decision making in the region.

\section{RESEARCH METHODOLOGY}

The researcher decided to formulate a methodology called Infosye Research Framework that would enable the actualization the research goal. Infosye Research Framework comprise of five distinctive stages which includes Description of Existing System, Challenges of the Existing System, Propose System Solution, Propose System Architectural Design and Recommendation. This stages are executed in a chronological order, whereby the first stage most be completely executed before the next stage until the entire stages are executed completely.

The first stage enables us to analyze and describe the characteristics of the existing system under study. That of the second stage gives an overview of the challenges of the existing system by stating the cause of these flaws. The third stage enables the researcher to propose a system solution that would resolve drastically the challenges of existing system. Information on how the proposes system will resolve the challenges are discussed at length at this stage. That of the fourth stage consist of the propose system design, showcasing how it would enhance the performance of the existing. The fifth stage enable the researcher to make some recommendation to an audience who may be interested in the propose system solution. See Diagram below for more insight. 


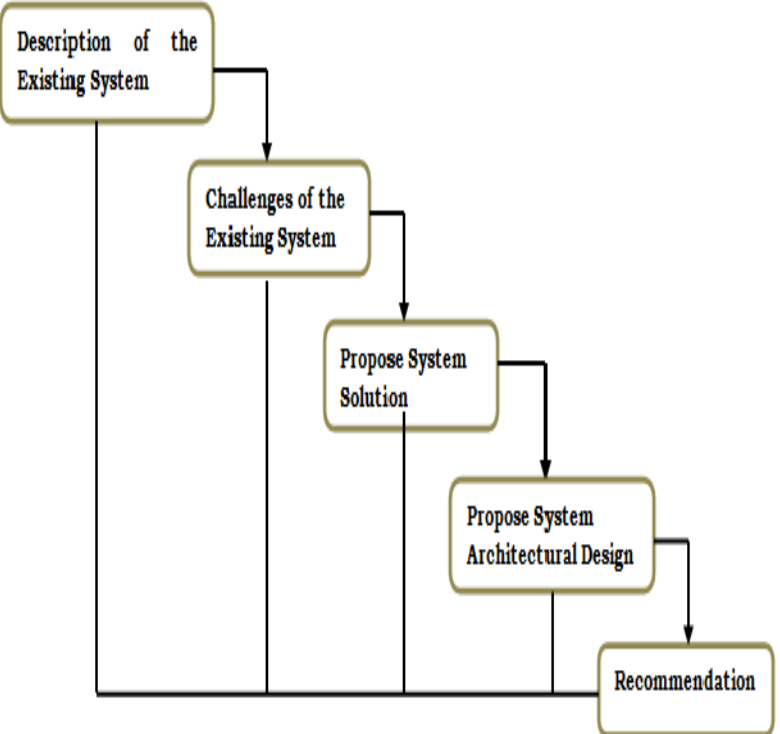

Figure 1: Infosye research framework

\subsection{Description of the Existing System}

A quarter of the world's births take place in West African countries where there are no complete civil registration system at all. Most of them uses corrected statistics, and in other cases predicted statics. Corrected statistics are based on survey data, adjusted in various ways to deal with missing data bias and different data collection methods predicted [32]. Statistics are presented for around one third of the countries with no recent, nationally representative surveys. Predicted child mortality birth and death estimates are generated by a statistical model, implemented in most West African countries.

Another method used for estimating child birth and death rate is called Sample Registration System. It is used to conduct a large scale demographic survey across the communities in the country. Births and Deaths within the sample population are constantly recorded by an enumerator. Another existing system for predicting child mortality birth/death rate is called Regression Technique. [34] employ Regression technique in their research study to predict child birth and death rate in Nigeria. This technique works by relying on data feedback from survey conducted either with questionnaire or observation across the communities in some parts of these countries. These data feedback are then estimated and analyze using a mathematical method called Regression Technique.

\subsection{Challenges of the Existing System}

Because of the uncertainty surrounding estimates derives from statistical model, predicted values are not appropriate for monitoring trends [22]. In order words, it is elusive to determine the estimates number of birth/death occurred in these developing countries. Leading to lack of accurate data that could be use to predict and monitor the trends as a preventive measure to curtail it. Regression technique relies heavily on feedbacks. Data collated from feedback are not validated to be accurate due to the fact that it is prone to human errors during data collection exercise. According to [34], the quality of Sample Registration System has also been questioned due to its flaws. This is due to the fact it is limited to a specific region. In order words, it only covers a nationally representative sample of the population rather than the population as a whole.

\subsection{Propose System Solution}

There are two possible Desktop Grid middleware Model that can be use to resolve the issues discussed which are called push and pull model. Push model is an approach that relies on publishes, subscribes and distributed paradigm. The publisher sends events and messages to an unknown group of participants [32]. The pull model is based on the request and response paradigm. This approach is typically used to perform data polling. The push model is the researcher's choice due to its efficiency, "no network or hardware failures, no disk space shortage and no service failure e $\mathrm{c}$ " [25].

The propose system would comprise of five work stations (Personal Computer System) namely Dispatcher, worker A, worker $\mathrm{B}$, worker $\mathrm{C}$ and worker $\mathrm{D}$. the dispatcher will initiate connections to the four workers. If connection is successfully established, the dispatcher will then splits job (to compute the total number of child mortality birth and death rate within a specific period e g. 2015 to 2020) into fragments and send these fragments of jobs to the four workers to execute. Each of these workers is expected to execute the job allocated to them independently without relying on one another. Upon completion of the fragmented jobs by each worker, are forward back to the dispatcher which in turn relay the completed job to Simul8.

The execution process i.e. how the workers are executing their task will be captured live with the aid of Simul8 software application and the expected estimated result (total estimate amount of child mortality birth and death). In other worlds, all the processes of the Desktop Grid middleware would be simulated and displayed by Simul8. Simlu8 will continuously present number of child that would be put to birth and the number of child that would die after birth, in respect to the period of time indicated. With this system in place, medical personnel can accurately predict in split seconds the total number of child to be put to birth or that would die after birth within a stipulated period of time in (e g. from 2015 to 2020). See Propose System Design below for more insight.

\subsection{System Architectural Design}

The diagram below showcase the design of the propose system solution. It comprise of different components and how these components are related together.

The Job Portal is a sub class or a special method of the Dispatcher. Its responsibility is to enable the entry of number of child birth and death per hour and the estimated timeframe (e g. 2015 to 2030). The job to be executed is the computation of the estimated total number of child birth and death rate between 2015 and 2030. The job is then divided into fragments and dispatched to the workers to execute. Each worker processes its own job fragment and then returns the result back to the dispatcher. Immediately the workers receive jobs from the dispatcher, Smul8 is triggered. The responsibility of Simul8 is to display computation process of the job and then present the estimated total number of child birth and death rate within the specified timeframe (2015 to 2030). Other information relating to the computational process is also simulated by Simul8. The schema at the top left corner of the design indicates the interactions between the dispatcher and the workers. With this system in place, prediction of child mortality birth and death rate within a period of time (for instance, from 2015 to 2050) can be 
achieved in split seconds or minutes. Because of the computation process will be executed by the combination of processing power of four computer systems. In order words, Simul8 process is speed up by the computational power of the combined PCs (Workers) to achieve the expected result.

The main benefit of the propose system is that, it will drastically reduce time and difficulty that are associated with complex computation. For instance, if we want to find out the

estimated total number of child mortality birth and death rate in West African region from 2016 to 2096, the system can actually speed up the computational process and the result obtained in seconds or minutes. Also, the propose system is fault tolerant. The breakdown of one worker cannot halt the execution process. Peradventure one of the worker breakdowns during process execution, its job fragment will be re-allocated to the other available workers to be processed and the desired result will also be achieved within a limited time. See architectural model below for more insight.

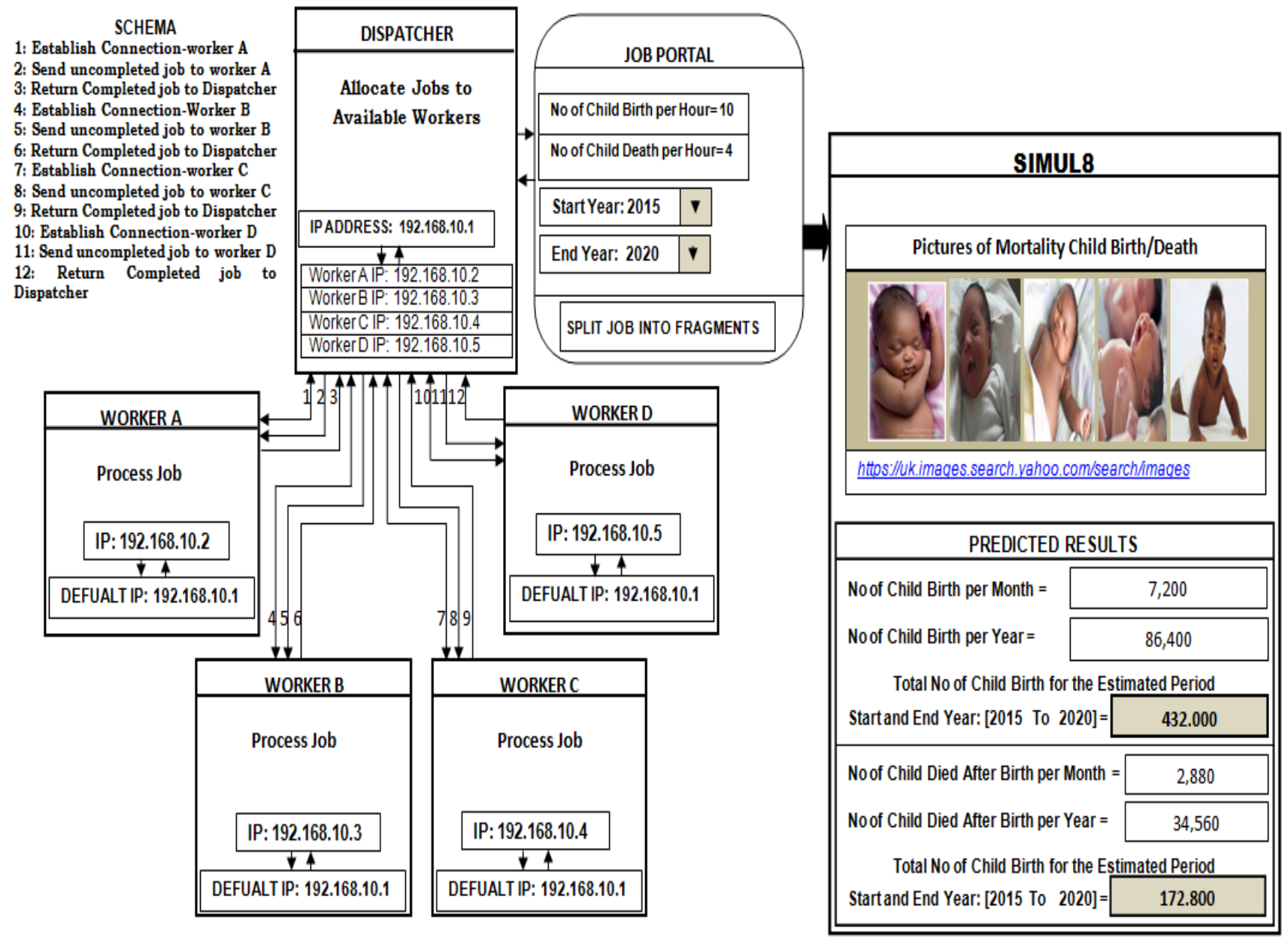

Figure 1.2: Proposed system architectural design

\subsection{Recommendation}

Clinicians, Government Agencies, United Nations interagency group for Mortality Estimation (UNIGME), World Health Organization, UNICEF, and other humanitarian NGOs across West African State should invest or acquire the working model of the proposed system. As it will go a long way to reduce complex computational stress drastically, thereby providing estimated results (total number of Child Mortality Birth and Death rate within specified period of time) as quick as possible. The result achieved will enable the efficient monitoring and control of the prevalent Child Mortality Birth and Death rate in the West African region. Also, the propose system could be acquire by other sub regional countries around the World especially developing countries in the Asian continents and its environs. These agencies (Clinicians, Government Agencies, United Nations Inter-Agency Group for Mortality Estimation (UNIGME) e $t$ c) tends to benefit tremendously from the propose system due to its processing power and robustness to execute complex computational task.

In conclusion, developing such complex computational system is elusive. However, the researcher as formulated a model that may enable the implementation of the proposed system solution. Prior to the research study, challenges were encountered. One of these challenges faced is the acquisition of Simul8 Software Application to ease the smooth simulation of the process under study (Prediction of Child Mortality 
Birth/Death rate in West Africa). However, the process was simulated with the tools that are available to the researcher. Other challenges encountered are lack of computational infrastructure and research materials such as articles e $\mathrm{t} c$.

In the future, the researcher intends to develop a working model of the proposed system solution. The working model would be implemented in a way that the grid middleware will consists of numerous workers. In order words, it will not be restricted to only four workers as illustrated in the design model, but will comprise of unlimited workers. Actual Simul8 software package will be employed to simulate the computational process as well as displaying expected results simultaneously.

\section{REFERENCES}

[1] Ali E. El-Desoky, Hishmam A. Ali, Abdulrahman A. Azab (2007). " Apure peer-to-peer Desktop Grid Framework with Efficient Fault Tolenrance", International Conference on Computer Engineering and Systems, Pages 346-352.Cairo, Egypt.

[2] Anu Maria (1997). "Introduction to Modelling and Simulation", Proceedings of the 1997 Winter Simulation Conference, http://www.informssim.org/wsc97papers/0007.PDF, [Accessed Date: 90-052015].

[3] Bernard Lapeyre (2007). "Introduction to Monte-Carlo Methods", http://cermics.enpc.fr/ bl/Halmstad/montecarlo/lecture-1.pdf [Accessed Date: 12-05-2015].

[4] Cicotti P., Taufer M. And Chein Andrew A. (2005). "A performance Monitoring Tool for Sanbox-Based Desktop Grid Platforms", Journal of Supercomputing, Volume 34. Issue No 2, Page 113-133

[5] Daniel Minoli (2005). "A network Approach to Grid Computing. United States of America, First Edition: Wiley Press.

[6] Daniel W. McGregor \& Matthew J. Cain (2009), An Introduction to SIMUL8, http://www.mang.canterbury.ac.nz/courseinfo/msci/msci 480/mcgregor_cain2004/webpage/simul8.pdf [Accessed Date: 14-05-2015].

[7] Danzhen You, Phillip Bastian, Jingxian Wu and Tessa Wardlaw (2013). "Levels \& Trends in Child MortalityEstimates Developed by the UN Inter-agency Group for Child Mortality Estimation", http://www.childinfo.org/files/Child_Mortality_Report_2 013.pdf [Accessed Date: 14-05-2015]

[8] David Krahl (2003). "Extend: An Interactive Simulation Tool", Proceedings of the 2003 Winter Simulation Conference, http://informs-sim.org/wsc03papers/023.pdf [Access Date: 09-05-2015]

[9] Deborah Balk, Tom Pullum, Adam Storeygard, Fern Greenwell and Melissa Neuman (2003). "Spatial Analysis of Childhood Mortality in West Africa" http://www.dhsprogram.com/pubs/pdf/SAR5/GS1.pdf [Accessed date: 14-05-2015]

[10] Demir, O. Head, M.R. Ghose, K and Govindaraju M (2005). "Protecting Grid Data Transfer Services with active Network Interfaces". The $6^{\text {th }}$ IEEE/ACM International Workshop, Page 8, United State Of America.
[11] Derrick Kondo, Andrew A. Chien and Henri Casanova (2004). "Resource Management for Rapid Application Turnaround on the Enterprise Desktop Grids", Conference on High Performance Networking and Computing; Page 17, Washington United State of America.

[12] F. Breitenecker and I. Troch (2002). "Simulation Software - Development and Trends", Control Systems, Robotics and Automation - Vol. 5, http://www.eolss.net/sample-chapters/c18/e6-43-0707.pdf, [Date Accessed: 09-05-2015]

[13] Franco Travostino, Joe Mambretti and Gigi KarmousEdwards (2006). Grid Networks, United States of America, First Edition; John Wiley.

[14] Ian Foster and Carl Kesselman (2004). The Grid 2. United States of America, Second Edition; Morgan Kaufmann.

[15] James F. Kurose and Keith W. Ross (2001), Computer Networking, First Edition. United States of America, Addison Wesley Longman.

[16] Jeremy J. S. B. Hall (2011). Types of Simulations, http://www.simulations.co.uk/SimulationDesign/Types\% 20of\%20Simulations.pdf [Accessed Date:12-05-2015]

[17] Jim Shalliker \& Chris Ricketts (2002), “An Introduction to SIMUL8 Release Nine", http://www.wirtschaft.fhdortmund.de/eurompm/bilbao/S8intro.pdf , [Accessed Date: 12-05-2015]

[18] Joseph Nnamdi Mojekwu and Uche Ibekwe (2012). "Maternal Mortality in Nigeria: Examination of Intervention Methods", International Journal of Humanities and Social Science, Vol. 2, No. 20, http://www.ijhssnet.com/journals/Vol_2_No_20_Special _Issue_October_2012/13.pdf [Accessed Date:14-052015]

[19] Joshy Joseph and Craig Fellestien (2004), Grid Computing. United State of America, First Edition, IBM Press.

[20] Kieran Concannon, Mark Elder, Kim Hindle, Jillian Tremble and Stanley Tse (2007). "Simulation Modelling With SIMUL8", http://www.simtech.hu/_data/VFS_6084183539ff1c826d a47589a021838c.pdf, [Accessed Date: 13-05-2015].

[21] Kieran H. Concannon, Kim I. Hunter and Jillian M. Tremble (2003). "SIMUL8-Planner Simulation-Based Planning and Scheduling, Proceedings of the 2003 Winter Simulation Conference, http://informssim.org/wsc03papers/188.pdf, [Accessed Date:14-052015]

[22] Leontine Alkema, Fengqing Chao, Danzhen You, Jon Pedersen and Cheryl C Sawyer (2014). "National, regional, and global sex ratios of infant, child, and under5 mortality and identifi cation of countries with outlying ratios: a systematic assessment", Volume: 2, www.thelancet.com/lancetgh, [Accessed Date: 25-052015]. 
[23] M. P. Bekakos, G. A. Gravvanis and H. R. Arabnia (2006), Grid Technologies. Great Britain, First Edition, WIT Press

[24] Marc I. Kellner, Raymond J. Madachy and David M. Raffo (1998). "Software process simulation modeling: Why? What? How?", The Journal of Systems and Software Vol No:46 ,PPt 91-105 http://sba.pdx.edu/faculty/davidr/draccess/WEB/publicati ons/JOURNAL/JSS-what-why-how.pdf [Accessed Date: 10-05-2015]

[25] Mustafee N. And Taylor J. Simon (2006). "Using a Desktop Grid to support simulation modelling", $28^{\text {th }}$ International Confernce on Information Technology Interfaces; Pages 557-562. Cavtat, Croatia.

[26] Naqib Daneshjo (2011). "Computer Modeling and Simulation", Proceedings of 2011 International Conference on Optimization of the Robots and Manipulators, http://www.ecosim.it/wpcontent/uploads/2013/11/Daneshjo2011.pdf [Accessed Date: 18-05-2015].

[27] Nicolae Zoran (2008). "A Desktop Grid Computing Approach for Scientific Computing and Visualization", Doctorial Thesis, Norwegian University of Science and Technology, http://www.idi.ntnu.no/research/doctor_theses/zoran.pdf [Accessed Date: 28-04-2015]

[28] Ogunjimi Lucas Olusegun, Ibe, Rosemary Thomas and Ikorok Maria Micheal (2012). "Curbing maternal and child mortality: The Nigerian experience", International Journal of Nursing and Midwifery Vol. 4(3), pp. 33-39.

[29] Onur Özgün, Yaman Barlas (2009). "Discrete vs. Continuous Simulation: When Does It Matter?", Proceedings of the 27th International Conference of The System Dynamics Society, July 26 - 30, 2009, Albuquerque, NM, USA

[30] Population Horizons (2014). Infant Mortality and Fertility, http://collen.ageing.ox.ac.uk/wpcontent/uploads/2014/07/Factsheet_5.pdf , [Accessed Date: 23-04-2015].

[31] Population Horizons (2014). Infant Mortality and Fertility, http://collen.ageing.ox.ac.uk/wpcontent/uploads/2014/07/Factsheet_5.pdf ， [Accessed Date: 23-04-2015].

[32] Population Reference Bureau (2014), Child and Maternal Mortality, http://www.prb.org/Publications/Datasheets/2014/2014world-population-data-sheet/data-sheet.aspx, [Accessed Date: 24-03-2015].

[33] Population Reference Bureau (2014), Child and Maternal Mortality, http://www.prb.org/Publications/Datasheets/2014/2014world-population-data-sheet/data-sheet.aspx, [Accessed Date: 24-03-2015].

[34] Robert E Black, Saul S Morris, Jennifer Bryce (2003). "Where and why are 10 million children dying every year?", Lancet Vol 361: PPt 2226-34, http://www.cfwshops.org/download/child_survival.pdf, [Accessed Date: 14-05-2015]
[35] Roger Mchaney (2010). "Understanding Computer Simulation", $\quad$ http://imsu.edu.ng/site/cmp/19.pdf [Accessed Date: 07-05-2015].

[36] Ruth Thomas (2003), What Are Simulations? - The JeLSIM Perspective, http://www.jelsim.org/resources/whataresimulations.pdf , [Accessed Date:18-05-2015]

[37] Samik Raychaudhuri (2008). Introduction to Monte Carlo Simulation, Proceedings of the 2008 Winter Simulation Conference, http://informssim.org/wsc08papers/012.pdf, [Accessed Date:13-052015].

[38] Saul S Morris,1 Robert E Black and Lana Tomaskovic (2003). "Predicting the distribution of under-five deaths by cause in countries without adequate vital registration systems", International Epidemiological Association, Vol 32, Issue $\quad$ No 6 http://ije.oxfordjournals.org/content/32/6/1041.full.pdf + h tml [Accessed date: 14-05-2015]

[39] SungJin Choi, MaengSoon Baik and ChongSun Hwang (2004). "Volunteer Availability Based Fault Tolerant Scheduling Mechanism in Desktop Grid Computing Environment", International Symposium on Network Computing and Applications, Page 727-235, Seoul Republic of Korea.

[40] T.O Olatayo1 and N.O Adeboye (2013). "Predicting Population Growth through Births and Deaths Rate in Nigeria", Mathematical Theory and Modelling, Vol.3, Issue No.1. http://www.iiste.org/ [Accessed Date:14-052015]

[41] Shmuel Ur, Elad Yom-Tov, and Paul Wernick (2007). "An Open Source Simulation Model Today or forever", Journal of Simulation Vol No 1-14, http://citeseerx.ist.psu.edu/viewdoc/download?doi=10.1. 1.510.3519\&rep=rep1\&type=pdf [Accessed Date: 10-052015]

[42] Uk B., Taufer M., Stricker T., Settanni G., Cavalli A., and Casfisch A (2003). "Combining task and data parallelism to speed up protein folding on desktop grid platform", Proceedings of the $3^{\text {rd }}$ IEEE/ACM International Symposium on Cluster Computing and Grid; Page 240-247, CA, United State of America

[43] UK.Images (2015). "Images of Newly Born African Child", https://uk.images.search.yahoo.com/search/images;_ylt= A9mSs2dmX2hVOYwAGMBLBQx.; ylu=X3oDMTBy ZmVxM3N0BGNvbG8DaXIyBHBvcwMxBHZ0aWQD BHNIYwNzYw--

? $\mathrm{p}=$ Newly+Born+African+Child \&fr=yfp-t -203 [Accessed Date: 29-05-2015]

[44] Unicef (2015), Monitoring the Situation of Women and Children, http://data.unicef.org/child-mortality/underfive, [Date Accessed: 24-03-2015]

[45] Vlatka Hlupic and Vesna Bosilj-Vuksic (2004), "Business Process Modelling Using SIMUL8", Proceedings 16th European Simulation Symposium, http://www.scs-europe.net/services/ess2004/pdf/bus48.pdf [Accessed Date: 14-05-2015] 